the patients at different age ranges. We created 3 cohorts with different age ranges at onset of disease. Patents aged less than 5 years (Group1), 5-10 years (Group2) and over 10 years (Group3) at the time of diagnosis of the first non-Raynaud involvement of jSSc.

Results: Up till now 88 patients were enrolled,14 patients (15\%) in Group1, 22 (25\%) in Group2 and $52(59 \%)$ in Group3. Diffuse subtype occurred in $71 \%$ in Group1, in $82 \%$ in Group2 and in 65\% in Group3. Most patients were Caucasian. Disease duration at time of inclusion into the cohort was 3.9 years in Group1, 4.9 years in Group2 and 2.2 years in Group3. ANA positivity was 57\% in Group1, $77 \%$ in Group2 and $86 \%$ in Group3. Anti-scl 70 was around 30\% in all groups. Anti-Centromere positivity was 7 to $10 \%$. Mean modified skin score was 12.4 in Group1, 16.5 in Group2 and 15.9 in Group3. Raynaud Phenomenon occurred in 85 to $95 \%$ of the patients. History of active or inactive ulceration occurred in $57 \%$ in Group1, 62\% in Group2 and 43\% in Group3. Decreased FVC under 80\% occurred in $43 \%$ in Group $1,32 \%$ in Group2 and $30 \%$ in Group3. Pulmonary hypertension occurred in $7 \%$ in Group1 and in $10 \%$ in Group3. No renal hypertension was observed. Urinary sedimentary changes occurred in $7 \%$ in Group 1 and in $10 \%$ in Group3. Gastrointestinal involvement occurred in 21\% in Group1, 45\% in Group2 and $27 \%$ in Group3. Musculoskeletal involvement occurred in 58 to $64 \%$. Patient global disease activity (VAS $0-100$ ) was 42.8 to 47.9 . Patient global disease damage (VAS 0-100) was 39.6- to 45.0. Physician global disease activity (VAS $0-100$ ) was 35.4-40.0. Physician global disease damage (VAS 0-100) was 37.1 in Group1, 41.3 in Group2 and 27.7 in Group3.

Conclusions: It seems to be that patients, with onset of the disease in younger age have more severe disease as patients with disease onset after the age of 10 years. We need more patients in our cohort to gain more sufficient data to prove our preliminary observation.

Disclosure of Interest: None declared

DOI: 10.1136/annrheumdis-2017-eular.3419

\section{AB0958 PROPOSAL FOR A JUVENILE SYSTEMIC SCLEROSIS RESPONSE INDEX (JSSCRI): RESULT OF THE CONSENSUS MEETING IN HAMBURG. GERMANY 11TH OF DECEMBER 2016}

I. Foeldvari ${ }^{1}$, D.F. Furst ${ }^{2}$, J. Anton ${ }^{3}$, E. Baildem ${ }^{4}$, M. Blakley ${ }^{5}$, T. Constantin ${ }^{6}$, P. Costa Reis ${ }^{7}$, M. Curran ${ }^{8}$, M. Cutolo ${ }^{9}$, C. Denton ${ }^{10}$, K. Fligelstone $^{11}$, B. Hinrichs ${ }^{12}$, F. Ingegnoli ${ }^{13}$, A. Kienast ${ }^{1}$, D. Němcova ${ }^{14}$, C. Pain ${ }^{4}$, C. Pilkington ${ }^{15}$, V. Smith ${ }^{16}$, D. Khanna ${ }^{17} .{ }^{1}$ Hamburg Center for Pediatric and Adolescent Rheumatology, Am Schön Klinik Eilbek, Hamburg, Germany; ${ }^{2}$ UCLA, Los Angeles, United States; ${ }^{3}$ Sant Joan de Déu Hospital, Barcelona, Spain; ${ }^{4}$ Alder Hey Children's Foundation NHS Trust, Liverpool, United Kingdom; ${ }^{5}$ Indiana University, Indianapolis, United States; ${ }^{6}$ Semme/weis University, Budapest, Hungary; ${ }^{7}$ Hospital de Santa Maria, Lisbon, Portugal; ${ }^{8}$ Northwestern University, Chicago, United States; ${ }^{9}$ University of Genova, Genova, Italy; ${ }^{10}$ Royal Free Hospital; ${ }^{11}$ FESCA, London, United Kingdom; ${ }^{12}$ Practice Buchholz, AMS, Hamburg, Germany; ${ }^{13}$ University of Milan, Milano, Italy; ${ }^{14}$ Department of Pediatrics and Adolescent Medicine Charles University, Prague, Czech Republic; ${ }^{15} \mathrm{GOSH}$, London, United Kingdom; ${ }^{16} \mathrm{GU}(H)$, Ghent, Belgium; ${ }^{17}$ Univ Michigan, Michigan, United States

Background: Juvenile Systemic Sclerosis (jSSc) is an orphan disease. There is increasing interest in testing novel therapies in the management of fibrotic diseases. Therefore, it is very important to develop a Response Index for jSSc (JSScRI) to distinguish effective therapies from placebo. In 2014 at the 1st JSScRI Consensus Meeting in Hamburg, following two rounds of a Delphi process, a proposal for domains and items for a JSScRI were made. In 2016, the 2nd JSScRI Consensus Meeting was held in Hamburg, Germany.

Objectives: To conduct a Nominal Group Technique (NGT) and select potential core data set items that could change as outcome measures) that will be incorporated in the development of a JSScRI.

Methods: Before the 2nd JSScRI Consensus Meeting, the items from the 1st JSScRI Consensus Meeting (2014) were scored via Email, in a Delphi by the participants of the current meeting. Participants included 14 experts in adult and juvenile SSc and a patient partner. During the subsequent face to face NGT meeting, moderated by DEF, and the items were scored anonymously by the participants after a nominal group discussion. The domains and items were scored regarding their importance for 1 year clinical trial from 1 (not relevant at all) to 9 (most relevant). A priori, it was agreed by the participants that the goal of the NGT was to exclude items that: 1 . Are not feasible and 2. do not represent a changeable outcome measure even though they may represent the impact of disease on quality of life, vocational or recreational activity. Items with a median score of $<4$ in which greater than $1 / 3$ of participants scored $1-3$ [despite the item having a median score $>3$ ] were excluded.

Table 1. Assessment of the Activity of the Musculoskeletal domain

\begin{tabular}{lcccc}
\hline & Whole Group & $1-3$ & $4-6$ & $7-9$ \\
\hline 1) Swollen joints & 7 & 0 & 1 & 12 \\
2) Limited range & 7.5 & 1 & 1 & 10 \\
3a) MMT & 8 & 0 & 1 & 11 \\
3b) CMAS & 7 & 4 & 1 & 7 \\
4) Presence of tendon friction rub & 7 & 1 & 2 & 10 \\
5a) CK & 8 & 1 & 1 & 11 \\
5b) Aldolase & 7 & 3 & 3 & 7 \\
\hline
\end{tabular}

Results: Seventy-one items in 13 domains were scored. Six items were not scored as they were felt to not represent an outcome measure or were non-feasible and six items received a median score less then 4 .

Table 1 provides an example of the musculoskeletal domain with the median scores for different outcome measures.

Conclusions: In a rigorous, NGT consensus meeting, some item reduction for the JSScRI was achieved. Items will be tested in a prospective way in the patients of the inception cohort of juvenile SSc (www.juvenile-scleroderma.com).

Disclosure of Interest: None declared

DOI: 10.1136/annrheumdis-2017-eular.2862

\section{AB0959 EVALUATE THE CARDIOVASCULAR RISK THROUGH CAROTID INTIMA-MEDIA THICKNESS IN PATIENTS WITH JUVENILE IDIOPATHIC ARTHRITIS IN THE YOUNG ADULT AGE}

J.J. Bethencourt, L. Expósito, S. Bustabad. Rheumatology, Hospital Universitario de Canarias, Santa Cruz de Tenerife, Spain

Background: The relationship between inflammation and atherosclerosis has been demonstrated, so it is important to identify early markers of the disease. Ultrasound carotid intima-media thickness (CIMT) measurement is a noninvasive, consistent, validated technique used as a marker to identify subclinical arteriosclerotic disease

The long-term risk of cardiovascular disease in young adult patients with juvenile idiopathic arthritis (JIA) is unclear and there are no risk management guidelines for these patients.

Objectives: To assess whether there is an increase in CIMT in the young adult with a history of JIA and to relate CIMT with classic cardiovascular risk factors in these patients.

Methods: Observational and cross-sectional study. Follow-up patients from transitional care between 18 and 36 years old, with JIA diagnosis by ILAR classification. Filiations data, anthropometric variables and activity disease scores were collected.

We performed, prior informed consent, CIMT measurement by radiofrequency with Esaote MyLab 70XVG. Three measurements were performed on each carotid artery, according to the protocol of the American Society of Echocardiography. Results: Of the 20 patients, $17(85 \%)$ women and $3(15 \%)$ men. Subtype distribution was $8(40 \%)$ oligoarticular, 1 of them ANA negative; $8(40 \%)$ polyarticular being 4 seropositive and 4 seronegative; 1 systemic $(5 \%) ; 2(10 \%)$ psoriatic arthropathy and $1(5 \%)$ HLA B27 positive arthritis. $66.7 \%$ are with disease modifying drugs (26.7\% synthetic and $40 \%$ biological), while $33.3 \%$ do not have specific treatment.

The main variables studied are described in the attached table (Table 1).

In two previous independent studies conducted by Falaschi and Huang, CIMT was found to be $0.54 \pm 0.03 \mathrm{~mm}$ and $0.54 \pm 0.06 \mathrm{~mm}$ in 26 and 38 patients, respectively. From these results a mean and standard deviation of $0.54 \pm 0.05 \mathrm{~mm}$ were obtained. Using this last result as a control group, we compared it with the results of our series $(0.466 \pm 0.068)$, with a significant difference $(p<0.001)$.

In the statistical analysis, using the Rho Spearman, a significant correlation of the CIMT with the time of evolution of the disease in years $(r=0.579)$ and systolic blood pressure $(r=0.621)$ was observed at the level of 0.01 and with $C$ Reactive Proteine $(r=0.524)$ and BMI $(r=0.471)$ at the 0.05 level.

\begin{tabular}{lccccc}
\hline & N & Minimum & Maximum & Media & Typical deviation \\
\hline Age & 20 & 18 & 36 & 24,75 & 5,350 \\
Diagnosis age & 20 & 1 & 16 & 9,70 & 5,048 \\
Evolution time (years) & 20 & 5 & 27 & 15,00 & 6,720 \\
BMI (Kg/cm ${ }^{2}$ ) & 19 & 15,5 & 38,5 & 24,505 & 6,4347 \\
Abdominal perimeter & 20 & 58 & 126 & 81,33 & 19,392 \\
Waist-hip index & 20 & 44 & 135 & 90,40 & 26,009 \\
Systolic blood pressure & 20 & 96 & 130 & 114,05 & 7,258 \\
Diastolic blood pressure & 20 & 60 & 99 & 73,80 & 12,271 \\
Left CIMT & 20 & 394 & 634 & 464,15 & 54,788 \\
Right CIMT & 20 & 337 & 600 & 467,45 & 80,275 \\
HAQ & 17 &, 00 & 2,25 &, 3529 &, 59986 \\
VAS pain (mm) & 18 & 0 & 8 & 3,17 & 2,618 \\
CRP (mg/L) & 17 &, 3 & 57,7 & 9,419 & 14,6040 \\
DAS28 & 16 &, 97 & 3,97 & 1,8856 &, 88204 \\
\hline
\end{tabular}

Conclusions: The carotid intima-media thickness of patients with JIA were lower than the controls previously described in the literature, so we will complement this study with our population controls.

In addition to classic cardiovascular risk factors such as systolic blood pressure and BMI, there is correlation with the evolution time in years of the disease and $\mathrm{CRP}$, so that in transitional care programs we must the activity of the disease and insist on identification, control and follow-up of the classics factors associated with cardiovascular risk.

Disclosure of Interest: None declared

DOI: 10.1136/annrheumdis-2017-eular.4029 